\title{
33. The rise of supercapacitors: making electric vehicles as convenient as ordinary ones
}

\section{Claude Henry}

Harold Kroto, at the University of Sussex, UK, had discovered chain-like carbon molecules in interstellar gas clouds. Would it be possible to vaporize carbon on Earth to produce similar molecules and find out what they are exactly made of? To try to answer this question, Kroto headed to Rice University (Houston, Texas), where Robert Curl and Richard Smalley had a very powerful laser that could vaporize almost any known material. He didn't know that his visit would bring to the three of them a Nobel Prize in chemistry.

In a series of experiments performed in the autumn of 1985, they simulated the physicochemical reactions that take place in interstellar clouds by applying the power of their laser to the most common form of carbon, graphite. The results were stunning: hitherto unobserved on Earth, and altogether remarkable, carbon molecules were detected. Because of their size, they are usually called nanoparticles. Since then, lots of nanoparticles have been engineered from various physical elements, not only carbon; some of them are endowed with astonishing mechanical, electrical and chemical properties. As far as mechanical strength is concerned, it can be two orders of magnitude greater than it is for steel. Electrical properties beat those of previously known electrically efficient materials. The high level of chemical activity is linked to the high surface area to volume ratio that small size entails. In short, one might say that ordinary materials, when embodied at very small scales, display remarkable properties that cannot be anticipated at larger scales.

This has been the launching pad for the explosive expansion of nanotechnologies, with myriad research and development initiatives aiming at all sorts of applications, some useful, some detrimental. Would supercapacitors emerge as among the most useful ones?

As a battery, a supercapacitor is made of two electrodes both in contact with an electrolyte, often a liquid, selected for its capacity to conduct and store electricity. In a supercapacitor - this is the main difference with a battery - no 
chemical reaction takes place; storage is electrostatic. That creates advantages and disadvantages.

The main advantages are the following:

- Supercapacitors are able to charge and discharge in a matter of seconds, that is, much faster than batteries.

- They can run without significant degradation up to 1 million cycles (charge-discharge) instead of a few thousand.

- They are safer in so far as no explosion is to be feared and they contain far less harmful chemicals and toxic material.

- Rare earths - mostly mined in China at the cost of devastating pollution - do not enter their fabrication, except for relatively abundant cerium and lanthanum compounds.

- They are much lighter.

- They can operate within far larger temperature ranges, circa $-40^{\circ} \mathrm{C}$ to $+60^{\circ} \mathrm{C}$.

The main disadvantage is that supercapacitors currently commercialized cannot match batteries in terms of energy density, that is, in the ability of delivering energy at a regular pace for relatively long durations.

Being able to discharge quickly, supercapacitors in a vehicle can deliver bursts of power for acceleration. This is, for instance, the case in some Toyota models. PSA Groupe (formerly PSA Peugeot Citroën) integrates supercapacitors in stop-start fuel-saving systems. Mazda makes use of supercapacitors to capture and store energy during deceleration. In these functions, supercapacitors appear complementary to batteries. Complementarity might also manifest itself in a different way: progress is made in associating battery and supercapacitor, the latter assisting the former so that the time required to charge the battery is cut to minutes.

From complements to replacements? That doesn't look unrealistic any longer. A number of university and industry labs are working on new classes of either electrodes or electrolytes in supercapacitors in order to enhance their performance, particularly their energy density.

Astonishing results have been obtained using various advanced graphene-based nanomaterials - particularly bright grandchildren to Kroto, Curl and Smalley - as main components in electrodes (Li et al., 2019; Yang et al., 2017). Graphene derivatives have been selected not only for their exceptional electrical, thermal and mechanical properties - which translate into supercapacitor performances - but also for available inexpensive production processes. New electrolytes have also been engineered that can store energy more efficiently (Mao et al., 2019). 
Actual leaps in energy density have been achieved. "They put", anticipates Dexter Johnson (2015) in the Institute of Electrical and Electronics Engineers' magazine, Spectrum, "storage capacity parity with Li-ion batteries within reach. And when you have a supercapacitor that has the storage capacity of a Li-ion battery and the ability to charge in mere moments, all-electric vehicles might just be a lot more attractive". That would be good for the climate, and much more.

\section{REFERENCES}

Johnson, D. (2015), "Supercapacitors take a huge leap in performance", IEEE Spectrum, 28 May.

Li, Q., M. Horn and Y. Wang et al. (2019), "A review of supercapacitors based on graphene and redox-active organic materials", Materials, 12 (5), 703.

Mao, X., P. Brown and C. Červinka et al. (2019), "Self-assembled nanostructures in ionic liquids facilitate charge storage at electrified interfaces", Nature Materials, 18 (8), 1350-7.

Yang, H., S. Kannappan, A. Sumuthira Pandian and J.-H. Jang (2017), "Graphene supercapacitor with both high power and energy density”, Nanotechnology, 28 (44), 445401 . 